\title{
Clave para la identificación de las especies de escorpiones de Uruguay
}

\section{Key to the identification of scorpion species from Uruguay}

\author{
Toscano-Gadea, Carlos Antonio ${ }^{(1)}$ \\ ${ }^{(1)}$ Laboratorio de Etología, Ecología y Evolución, Instituto de Investigaciones Biológicas Clemente Estable, Uruguay. \\ Contacto: ctoscanogadea@gmail.com \\ Recibido: 17/3/2012 - Aprobado: 16/10/2012
}

\author{
$\underline{\text { Resumen }}$ \\ Este artículo presenta una clave para el reconocimiento de las especies de escorpiones halladas en Uruguay. Asimismo, se \\ brinda información sobre su comportamiento, biología, distribución e importancia médica. \\ Palabras clave: Taxonomía, escorpiones, Uruguay.

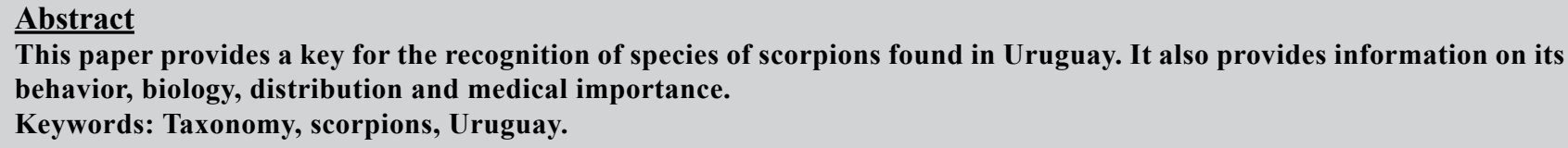

\section{Introducción}

A nivel mundial el Orden Scorpiones está compuesto por 16 familias y aproximadamente 1.600 especies (Fet et al., 2000). Los escorpiones son un grupo de antiguo linaje (existen registros de las primeras formas terrestres de hace aproximadamente 350 millones de años) y se encuentran distribuidos en todos los continentes excepto la Antártida (Mattoni, 2003).

A la fecha, en Uruguay se conocen tres familias, cuatro géneros y siete especies de escorpiones. De estas especies dos son endémicas de nuestro país, dos fueron introducidas por el ser humano, dos se reproducen de forma partenogenética y una posee importancia médica.

Si bien en Uruguay existen claves que permiten identificar a otros órdenes de arácnidos (Benamú, 2007; Montes de Oca y Pérez-Miles, 2009; Giuliani et al., 2011), no hay disponible hasta el momento una clave que permita identificar a las especies de escorpiones del país.

El objetivo de este trabajo es ofrecer las herramientas necesarias para la identificación de las especies de escorpiones presentes en
Uruguay. Para este fin se brinda una clave a nivel de especies y una breve descripción de las principales características de cada una, acompañada de fotos para su reconocimiento.

\section{Materiales y Métodos}

Para la confección de la clave se revisó el material depositado en la Colección Entomológica de la Facultad de Ciencias, Universidad de la República, Montevideo, y el material disponible en el Instituto de Investigaciones Biológicas Clemente Estable, Montevideo.

Los tamaños a los cuales se hace referencia consideran siempre valores promedio de ejemplares adultos, medidos desde el borde anterior del prosoma hasta el extremo del telson. Se excluyen en estas medidas los apéndices. La coloración está indicada para animales adultos y vivos, ya que pueden existir diferencias en ejemplares juveniles o con aquellos que se encuentran mantenidos en líquidos conservantes. Finalmente, se incorpora un glosario con los principales términos utilizados.

\section{Resultados}

\section{Clave de especies}

1.- Color general del cuerpo oscuro (castaño oscuro o

negro)

Color general del cuerpo claro (castaño

claro) 
Vesícula de color amarillo

3.- Vesícula con apófisis subaculear, cuerpo y pedipalpos de aspecto

delicados

Vesícula sin apófisis subaculear, cuerpo y pedipalpos de aspecto robusto

4.- Aspecto del cuerpo robusto, con variación en la coloración del negro al castaño. Pueden llegar a medir 50 o $70 \mathrm{~mm}$. Cara dorsal de la vesícula de los machos con una zona media glandular ubicada en una oquedad profunda, de color blanco intenso, menos visible en las hembras. Con 16 a 23 dientes pectíneos en cada peine. Cara ventral del segmento caudal V con carenas ventrales lateral (VL) y ventrales submedianas (VSM) formando un semicírculo, abierto en su parte posterior. Tarsos de las patas III y IV con tres pares de espinas. Quelíceros con un diente sub distal generalmente bien desarrollado

Bothriurus bonariensis (C. L. Koch 1842)

Coloración del cuerpo castaño oscura, con patas rojizas. Tamaño entre 40 y 50 mm. La glándula del telson en los machos es una leve depresión de color más claro, en las hembras solo se aprecia la coloración más clara. La vesícula algo más alargada que en la especie anterior. El telson es de color castaño oscuro. Con 13 a 17 dientes pectíneos en cada peine. Cara ventral del segmento caudal V con un semicírculo abierto en su extremo posterior, formado por las carenas VL y VSM que no llega a cerrarse, con una carena Ventral Mediana en forma de Y de aproximadamente 14 gránulos en el macho y 11 en la hembra. Hacia los costados de esta Y hay dos gránulos. Quelíceros con 2 dientes sub distales en el dedo móvil

Bothriurus rochensis San Martín 1964.

5.- Cuerpo de aspecto robusto, achatado, pinzas de los pedipalpos grandes y fuertes, con carenas, y con una lobulación en la cara dentada interna de los dedos. Metasoma de aspecto delicado, con segmentos muy delgados y cortos, terminado en una vesícula con coloración amarillo intensa. Posee entre 6 y 12 dientes pectíneos en cada peine. Tamaño entre 40 y $60 \mathrm{~mm}$ Euscorpius flavicaudis (De Geer 1778).

6.- Tamaño entre 40 y $50 \mathrm{~mm}$. Color del cuerpo castaño claro uniforme, con tres líneas algo más oscuras. Tubérculo ocular de color más oscuro. Cuerpo achatado, de aspecto grácil. Pinza de los pedipalpos con tibia y tarso delgados y largos. Presentan 12 a 17 dientes pectíneos en cada peine. Vesícula con apófisis sub aculear en su cara dorsal Tityus uruguayensis Borelli 1901.

Tamaño entre 40 y $70 \mathrm{~mm}$. El color del cuerpo varía entre el castaño claro y el castaño oscuro. Presenta tres bandas longitudinales de color castaño oscuro, fácilmente visibles. Tubérculo ocular muy oscuro. Presentan de 18 a 22 dientes pectíneos en cada peine. Vesícula con apófisis sub aculear en su cara dorsal. A diferencia de lo observado en T. uruguayensis, el extremo de la tibia y tarso de los pedipalpos y del telson poseen color negro Tityus trivitattus Kraepelin, 1898.

7.- Tamaño entre los 30 y $50 \mathrm{~mm}$. Cuerpo robusto, coloración amarillo clara con manchas oscuras. Metasoma con líneas ventrales submedianas. Tarso de las patas III con 5 a 7 pares de espinas, tarso IV con 6 a 7 pares. Presentan entre 13 y 16 dientes pectíneos en cada peine. Quelíceros con 2 dientes subdistales en el dedo móvil, el anterior más pequeño que el posterior. Pinzas de los pedipalpos de aspecto fuerte, sin carenas

Urophonius iheringii Pocock 1893.

Coloración del cuerpo castaño clara con manchas algo más oscuras. Cara ventral de coloración castaño oscura, con dos líneas claras que la atraviesan de forma longitudinal. Metasoma con una sola línea oscura ventral media. Tamaño entre 30 y $50 \mathrm{~mm}$. Vesícula de forma alargada y fina, con la cara dorsal sin excavación, pero con coloración levemente más clara (principalmente en el macho). Telson muy agudo. Presentan de 12 a 17 dientes pectíneos en cada peine. Cara ventral 
del segmento caudal V con las carenas VL y VSM, con una carena mediana

formada por 16 gránulos gruesos. Quelíceros con diente sub distal, aunque en

algunos casos pueden presentarse dos dientes vestigiales unidos en la base

Bothriurus buecherli San Martín 1963.

\section{Comentarios sobre las familias y especies de escorpiones de Uruguay}

\section{Familia Bothriuridae Simon, 1880.}

La familia Bothriuridae es predominantemente neotropical. Actualmente se conocen 12 géneros sudamericanos, dos géneros africanos y un género australiano - que también fue citado para La India (Lourenço, 1996)-, lo que sugiere un origen en el viejo supercontinente Gondwana (Lourenço y Monod, 2000; Prendini, 2003; Ojanguren-Affilastro, 2005). En Uruguay es la familia más diversa con cuatro especies descriptas, y otras entidades aún no descriptas. Son escorpiones de tamaño medio, con un periodo de actividad que puede ser estival o invernal, y que se encuentran distribuidos por todo el territorio nacional. Algunas de sus especies presentan hábitos sinantrópicos muy marcados (Toscano-Gadea, 2011a).

\section{Bothriurus bonariensis (C. L. Koch 1842) (Figura 1 y 2)}

Tamaño: Mide entre 50 y $70 \mathrm{~mm}$, los machos son algo más grandes que las hembras.

Coloración: Variable, dependiendo de si se lo encuentra al sur o al norte del Río Negro. Al sur su coloración es negra uniforme pero varía al castaño-rojizo si se lo encuentra al norte. A pesar de las diferencias en la coloración, por el momento se considera a estas distintas poblaciones como pertenecientes a una misma especie.

Diferencias entre los sexos: Dimorfismo sexual evidente. Los machos poseen una glándula ubicada en una hendidura o foseta en la zona dorsal de la vesícula que resulta fácilmente distinguible por su color blanco intenso (Figura 3). Posiblemente a través de esta estructura se segreguen sustancias que estimulen o apacigüen a la hembra durante el cortejo y la cópula (Peretti, 1997). Los machos además presentan los pedipalpos más globosos y la tibia y el tarso más cortos. En la cara interna, en la base del dedo móvil (inferior) del macho se observa una espina (Figura 4) que interviene en la sujeción de la pinza de la hembra durante el cortejo y cópula (Figura 5).

Las hembras poseen los pedipalpos más alargados, la tibia y el tarso largos y sin la espina de la cara interna del pedipalpo. Aunque se observa una coloración más clara en el lugar donde estaría ubicada la foseta, no existe una depresión profunda como la que está presente en los machos.

Hábitats: Viven tanto en ambientes naturales como modificados. Se ubican debajo de refugios criptozoicos: troncos caídos, piedras, cortezas de árboles y hojarasca, con elevada humedad y ausencia de luz (Costa \& Pérez-Miles, 1994; Toscano-Gadea, 2002). Presentan escasa sinantropía; existen eventuales registros de incursión dentro de la vivienda humana, pero es común encontrarlo en la periferia de la misma, donde se ubica debajo de restos de construcciones o pilas de leña. Muy abundante en praderas ganaderas donde se refugia debajo del estiércol vacuno y en los montes cultivados (San Martín, 1961). Excava refugios o galerías, que pueden llegar a medir varios centímetros de largo (San Martín, 1961).

Reproducción: Sexuada, las hembras tienen entre 40 y 50 crías una vez al año.

Peligrosidad: Se considera inofensivo para el hombre.

Actividad: Meses cálidos, desde finales de noviembre hasta finales de marzo. En el norte del nuestro país puede finalizar entrado abril. Nocturno.

Distribución: Está presente en Argentina, Brasil y Uruguay. En Uruguay está distribuido en todo el territorio nacional.

Comentarios: Carenas ubicadas en la cara ventral del segmento caudal V forman un semicírculo casi completo (Figura 6).

\section{Bothriurus rochensis San Martín 1965. (Figura 7)}

Tamaño: Entre 40 y $50 \mathrm{~mm}$. Las hembras son más pequeñas que los machos.

Coloración: Aspecto muy similar al de B. bonariensis (lo cual lleva a confusiones en su determinación). Cuerpo negro oscuro con extremidades de coloración castaño rojiza, lo cual permite su identificación (San Martín, 1965).

Diferencias entre los sexos: Dimorfismo sexual evidente. Los machos poseen foseta en la cara dorsal del telson (aunque menos profunda que en $B$. bonariensis), y pedipalpos globosos con la tibia y el tarso cortos. En la cara interna del palpo, en la base del dedo móvil se observa una espina. Las hembras tienen las tibias y tarsos de los pedipalpos más alargados y sin la espina característica de los machos.

Hábitats: Esta especie se encuentra tanto en ambientes naturales como modificados. Se ubica debajo de refugios criptozoicos, principalmente pedregales, hojarasca y troncos caídos (San Martín \& Gambardella, 1967; Toscano-Gadea, 1996). Muy abundantes en la franja costera del departamento de Rocha, en montes nativos o cultivados y aun en zonas anegables (Toscano-Gadea, 2011a). Excavan refugios o galerías pero sensiblemente más cortas que $B$. bonariensis. Presentan alta sinantropía, durante los meses cálidos es común hallarlos dentro de las viviendas humanas.

Reproducción: Sexuada, las hembras tienen un promedio de 40 crías una vez al año.

Peligrosidad: Se lo considera inofensivo para el hombre.

Actividad: Meses cálidos, desde noviembre hasta finales de marzo. Nocturno.

Distribución: Esta especie es endémica de Uruguay, presente en los departamentos de Maldonado, Lavalleja y Rocha.

Bothriurus buecherli San Martín 1963. (Figura 8)

Tamaño: Entre 30 y $50 \mathrm{~mm}$. Machos algo más grandes que las hembras. 
Coloración: Castaño clara con áreas depigmentadas en forma de manchas amarillas (San Martín, 1963). Las cuales forman franjas depigmentadas dorsales a lo largo del mesosoma.

Diferencias entre los sexos: Dimorfismo sexual menos evidente que los casos anteriores. Machos con glándula de la cara dorsal del telson poco visible y de coloración menos conspicua. Se mantiene el dimorfismo sexual observado en otras especies de Bothriurus. Los machos poseen los pedipalpos globosos, con la tibia y el tarso cortos. En la cara interna del palpo, en la base del dedo móvil se observa una espina. Las hembras tienen las tibias y tarsos de los pedipalpos más alargados y sin la espina presente en los machos.

Hábitats: En áreas naturales se ubica en refugios criptozoicos, principalmente de zonas serranas (Costa y Pérez-Miles, 1994; ToscanoGadea, 2002). Es de escasa sinantropía, aunque posee hábitos peri domiciliarios. Es posible encontrarlo en la cercanía de viviendas humanas en algunos balnearios de la Costa de Oro. No realizan galerías, sólo refugios rudimentarios.

Reproducción: Sexuada, las hembras tienen un promedio de 30 crías una vez al año.

Peligrosidad: Se lo considera inofensivo para el hombre.

Actividad: Meses cálidos, desde noviembre hasta finales de abril. Nocturno.

Distribución: Especie endémica de Uruguay. Presente en los departamentos de Montevideo, Canelones, Maldonado, Rocha y Lavalleja.

Urophonius iheringi Pocock 1893. (Figura 9)

Tamaño: Entre 30 y $45 \mathrm{~mm}$. Machos más grandes que las hembras.

Coloración: Clara, con manchas oscuras irregulares ubicadas en la cara dorsal del cuerpo y extremidades. Por su coloración a simple vista pueden confundirse con ejemplares de B. buecherli.

Diferencias entre los sexos: Dimorfismo sexual evidente. Los machos poseen una espina en la cara interna del pedipalpo, una pequeña depresión debajo de la misma y una serie de tres o cuatro gránulos accesorios, en la base de la cara interna del dedo fijo. Todas estas estructuras están ausentes en las hembras (Acosta, 1988).

Hábitats: No construye galerías, se ubica en la periferia de las rocas alisando el sustrato. Las hembras construyen cámaras de cría donde se refugian durante la gestación. Tiene hábitos peri domiciliarios. Existen escasos registros de su presencia dentro de la vivienda humana. Se ha adaptado a las plantaciones en montes cultivados (Pinos), donde se sitúa en la gruesa cobertura de hojarasca del suelo y dentro de los troncos en descomposición (Toscano-Gadea, MS en preparación).

Reproducción: Sexuada, las hembras tienen entre 30 y 40 crías una vez al año.

Peligrosidad: Se lo considera inofensivo para el hombre, aunque su aguijonazo es doloroso.

Actividad: Meses fríos, otoño a invierno, generalmente desde mayo a setiembre. Se propone que el período reproductivo invernal podría ser una adaptación para no competir por recursos con otras especies de escorpiones (Maury, 1968; 1969; Ojanguren-Affilastro, 2005). Nocturnos.

Comentarios: Como ya se dijo, el aspecto del cuerpo puede recordar a B. buecherli, pero el segmento $\mathrm{V}$ del metasoma y la vesícula de $U$. iheringi son más largas que en la otra especie.

\section{Familia Euscorpiidae Laurie, 1896.}

La familia Euscorpiidae se encuentra presente en Europa y América del Sur, donde fue introducida por el hombre. Se conocen entre cuatro y seis géneros (Fet et al., 2000). En Uruguay está presente solamente una especie.

Euscorpius flavicaudis (De Geer 1778). (Figura 10)

Tamaño: Entre 50 y $60 \mathrm{~mm}$. Macho de mayor tamaño que la hembra.

Coloración: Cuerpo negro homogéneo, con patas castaño claro. Sobresale el metasoma por la coloración amarilla de la vesícula (flavicaudis significa cola amarilla).

Diferencias entre los sexos: Dimorfismo sexual evidente. El macho posee pedipalpos más grandes y desarrollados, que poseen un lóbulo en la cara dentada superior del dedo móvil, que se corresponde a una hendidura en la cara inferior del dedo fijo. Las hembras poseen los pedipalpos menos desarrollados y las estructuras anteriormente descritas se encuentran muy poco desarrolladas.

Hábitats: Esta especie fue introducida al país por la importación de mercaderías, probablemente desde Europa, con el puerto de Montevideo como la posible vía de ingreso al país (Toscano-Gadea, 1998). En Montevideo se lo encuentra asociado a terrenos baldíos, donde se ubican en huecos entre ladrillos o bloques. Altamente sinantrópico, comúnmente ingresa a los domicilios. Registros recientes sugieren que esta especie está ampliando su zona de distribución en Montevideo (Toscano-Gadea, 2011b).

La distribución original de esta especie abarca el sur de Europa. También se halló en Inglaterra una población en el puerto de Sheernes, en Kent (Benton, 1991; 1993), donde podría haber colonizado de la misma forma en que lo hizo en Uruguay.

Reproducción: Sexuada, las hembras tienen una camada al año de aproximadamente 20 o 30 crías.

Peligrosidad: Se lo considera inofensivo para el hombre.

Actividad: Meses cálidos, de noviembre a finales de febrero. Nocturno.

Distribución: Departamento de Montevideo. 


\section{Familia Buthidae C.L. Koch, 1837.}

La familia Buthidae posee distribución cosmopolita, con especies en todos los continentes a excepción de la Antártida y Nueva Zelanda. Hasta el momento se conocen más de 70 géneros, dentro de los cuales se encuentra Tityus, que es exclusivamente americano e incluye a gran cantidad de especies de importancia médica para el ser humano (Fet et al., 2000; Flórez, 2001; Ojanguren-Affilastro, 2005). Muchas de las especies de este género tienen la capacidad de reproducirse partenogenéticamente, ya sea en forma obligada u opcional, lo que otorga un elevado potencial colonizador y las convierte en especies altamente exitosas en la invasión de nuevos ambientes. En el país existen dos especies de Buthidae, ambas pertenecientes al género Tityus.

Tityus uruguayensis Borelli 1901. (Figura 11)

Tamaño: Entre los 40 y $50 \mathrm{~mm}$. Machos y hembras poseen tamaño similar.

Coloración: Castaña, el cuerpo es algo más oscuro mientras que los apéndices y el metasoma son más claros. La coloración del cuerpo los hace casi totalmente crípticos contra el sustrato.

Diferencias entre los sexos: Dimorfismo sexual casi inexistente a simple vista. La lamela basal intermedia de los peines de las hembras es de aproximadamente el doble de tamaño que en los machos (Ojanguren-Affilastro, 2005). El primer diente pectíneo de los machos es algo más grande y globoso que en la hembra (Lourenço y Maury, 1985).

Hábitats: Habita tanto en zonas naturales como artificiales, debajo de piedras (ubicado en su cara ventral), troncos, materiales de construcción. De baja sinantropía, se registran escasas citas de su presencia dentro de la vivienda humana, pero puede tener hábitos peri domiciliarios. No practica galerías. Posiblemente se encuentre presente en todo el territorio nacional. En posición de reposo, los ejemplares mantienen el metasoma apoyado en el sustrato, enrollado y dispuesto al costado del cuerpo. Es común encontrar más de un ejemplar debajo de un refugio (Figura 12).

Reproducción: Esta especie es considerada partenogenética (Zolessi, 1985). Estudios actuales señalan la presencia frecuente de machos, por lo que la partenogénesis podría ser facultativa y la hembra recurriría a este tipo de reproducción bajo determinadas circunstancias (ToscanoGadea, 2001; 2008).

Características diagnósticas: En la vesícula poseen una estructura denominada "apófisis subaculear", la cual se asemeja a un segundo aguijón aunque algo más pequeño. Esta estructura es importante para su identificación (Figura 13).

Peligrosidad: Si bien el C.I.A.T. posee registros de accidentes causados por esta especie (Heuhs et al., 2002), presentaron escasa sintomatología. A pesar de ello y por pertenecer al género Tityus, esta especie debe considerarse potencialmente peligrosa.

Actividad: Meses cálidos, de enero a marzo. Nocturno.

Distribución: Uruguay, sur de Brasil (Río Grande do Sul) y Argentina (provincia de Entre Ríos).

Tityus trivitattus Kraepelín 1898. (Figura 14)

Tamaño: Entre 40 y $70 \mathrm{~mm}$. Machos y hembras poseen tamaño similar.

Coloración: Amarillo-castaña. El cuerpo tiene dorsalmente tres bandas longitudinales de color castaño oscuro. El aguijón y los dedos de las quelas se encuentran fuertemente pigmentados de color negro.

Diferencias entre los sexos: Dimorfismo sexual casi inexistente a simple vista. En los machos el segmento caudal V es ligeramente más globoso y la pinza es algo más robusta, y con una levísima lobulación en la cara dentada interna de los dedos que está ausente en las hembras (Ojanguren-Affilastro, 2005). Sin embargo, casi con seguridad las poblaciones presentes en Uruguay son sólo de hembras partenogenéticas.

Hábitats: Esta especie también fue introducida en Uruguay, pero en este caso desde Argentina. En Uruguay solo hay dos citas de su presencia: en un establecimiento rural y en las inmediaciones de la Plaza de Toros del departamento de Colonia (Toscano-Gadea, 2011a, b). Tienen hábitos peridomiciliarios y son de alta sinantropía. Este comportamiento y la composición de su veneno hacen que deba ser considerada peligrosa.

Reproducción: Especie con poblaciones partenogenéticas (Toscano-Gadea, 2005) y sexuadas (Maury, 1997). Las hembras viven entre cuatro y cinco años luego de llegar a adultas y son capaces de tener una camada de entre 20 y 30 crías por año.

Características diagnósticas: Al igual que lo observado en T. uruguayensis, esta especie posee una apófisis subaculear. En este caso, esta estructura es notoriamente más pequeña que el aguijón. Al igual que en el caso anterior, en posición de reposo mantienen el metasoma apoyado en el sustrato y dispuesto al costado del cuerpo (Figura 15).

Peligrosidad: Su veneno tiene acción neurotóxica, por lo cual debe calificarse como peligroso para el ser humano. En el norte de la República Argentina se han reportado varios casos fatales, sobre todo en niños pequeños (Maury, 1997).

Actividad: Principalmente durante los meses cálidos, de noviembre a marzo, aunque es posible encontrar ejemplares activos durante todo el año. Nocturno.

Distribución: Argentina, Uruguay, Paraguay y Brasil. 
Figura 1

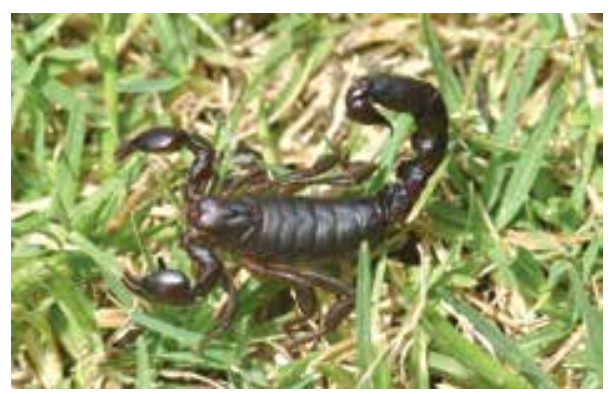

Figura 2

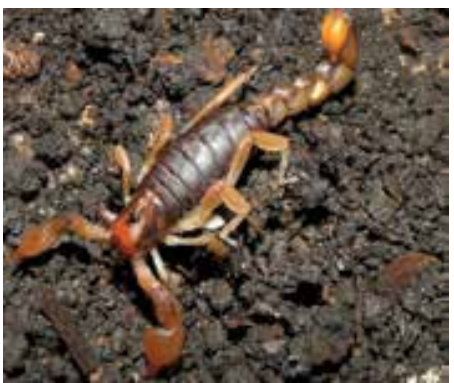

Figura 3

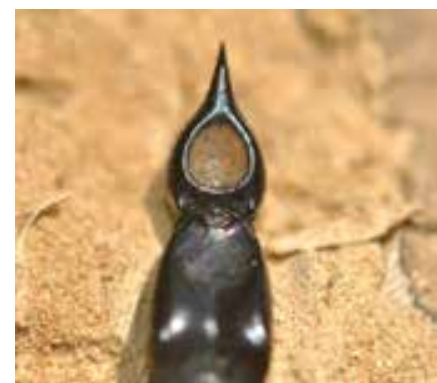

Figura 4

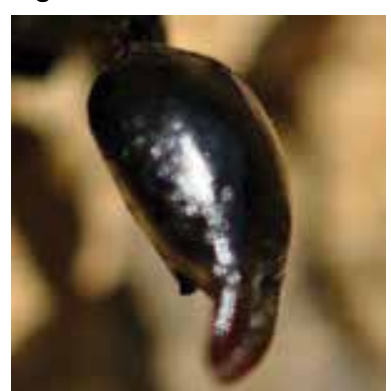

Figura 7

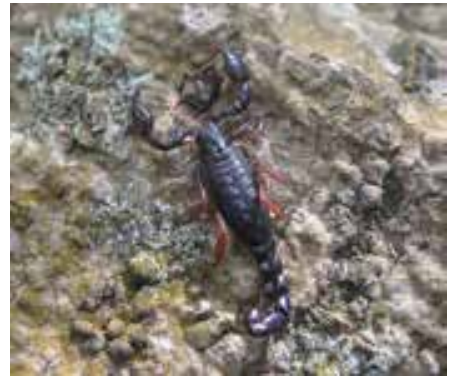

Figura 10

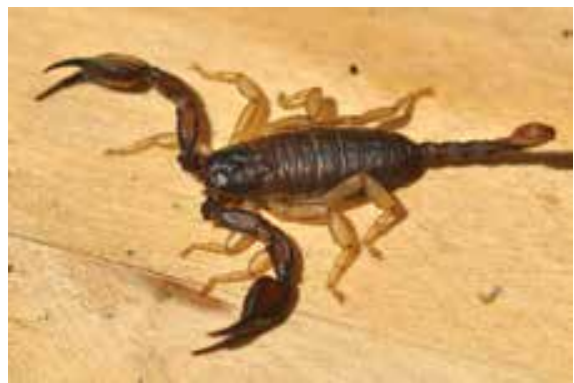

Figura 13

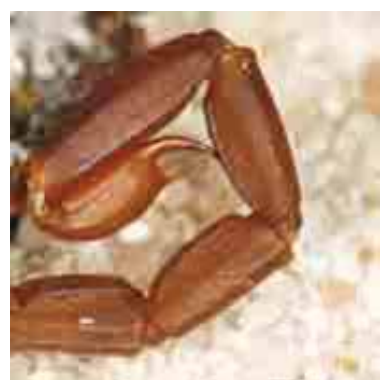

Figura 5

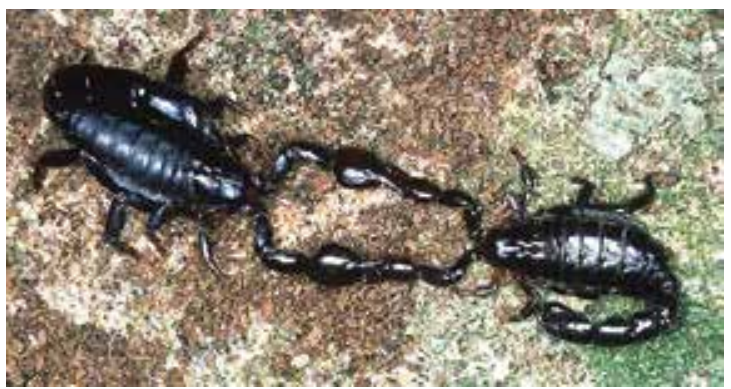

Figura 8

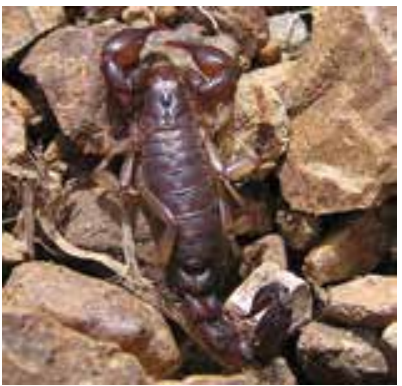

Figura 11

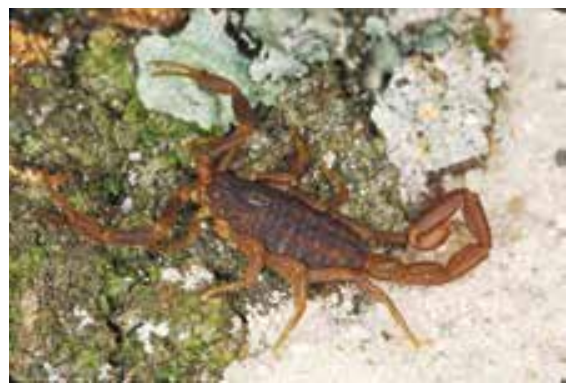

Figura 14

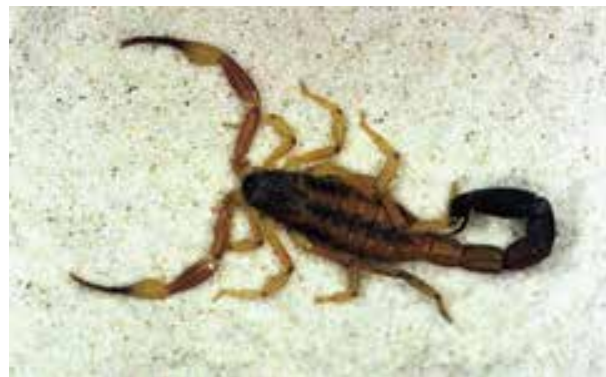

Figura 6

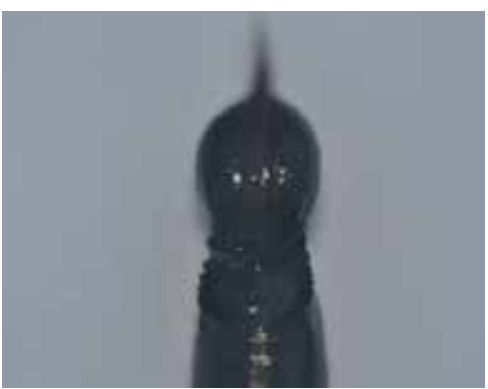

Figura 9

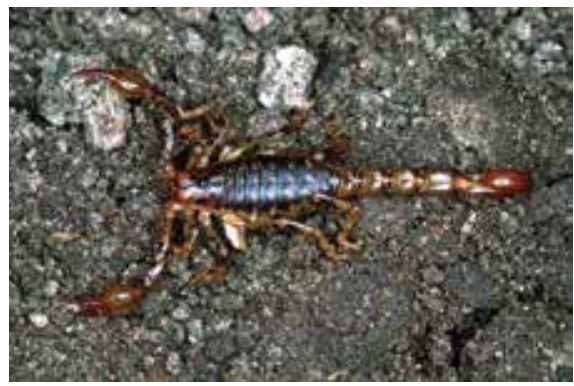

Figura 12

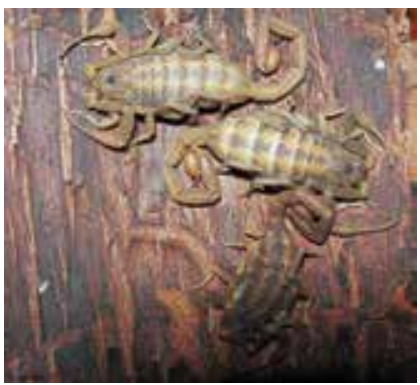

Figura 15

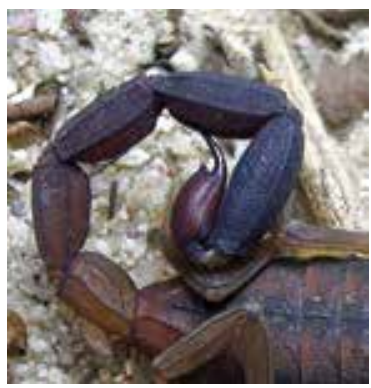

Figura 1: Macho de Bothriurus bonariensis. Coloración de los ejemplares del sur de Uruguay. / Figura 2: Macho de Bothriurus bonariensis. Coloración de los ejemplares del norte de Uruguay. / Figura 3: Detalle de la hendidura ubicada en la zona dorsal de la vesícula, de un macho de Bothriurus bonariensis. / Figura 4: Detalle de la espina ubicada en la cara interna del pedipalpo de un macho de Bothriurus bonariensis. / Figura 5: Macho y hembra de Bothriurus bonariensis durante la danza nupcial. Los pedipalpos del macho (izquierda) son más grandes que los de la hembra (derecha). / Figura 6: Carenas de B. bonariensis. Forman un semicírculo casi cerrado. / Figura 7: Macho de Bothriurus rochensis. / Figura 8: Macho de Bothriurus buecherli. / Figura 9: Macho de Urophonius iheringii. / Figura 10: Macho de Euscorpius flavicaudis. / Figura 11: Hembra de Tityus uruguayensis. / Figura 12: Hembras de Tityus uruguayensis juntas, debajo de un tronco caído. / Figura 13: Detalle del telson y la apófisis sub aculear en Tityus uruguayensis. / Figura 14: Hembra de Tityus trivittatus. / Figura 15: Detalle del telson y la apófisis sub aculear en Tityus trivittatus. 


\section{Glosario}

Apófisis subaculear: Se encuentra por debajo del aguijón y es relativamente similar en forma a éste. Su forma puede ser aguzada o roma según la especie y no inocula veneno. En Uruguay es una estructura presente solo en las especies de Buthidae.

Carena: Series ordenadas de gránulos, o elevaciones del tegumento, ubicados en el tegumento del escorpión. Su disposición tiene importancia en la identificación de las especies, particularmente en Bothriuridae.

Dientes pectíneos: Se encuentran en la cara ventral del cuerpo. Son estructuras que cumplen funciones sensoriales. A la estructura que agrupa a los dientes pectíneos se le da el nombre de peine o pecten.

Diente subdistal: Es el nombre del tercer diente (contando desde el extremo apical) ubicado en el dedo móvil de los quelíceros. Su número, forma y características tiene importancia en la identificación de las especies, particularmente en Bothriuridae, pudiendo haber uno o dos.

Dimorfismo sexual: Diferencias morfológicas entre machos y hembras de una misma especie.

Glándula del telson: Es un tejido glandular, generalmente ubicado en una depresión o foseta, de color claro y de profundidad variable. Se encuentra en la cara dorsal de la vesícula de varias especies de la familia Bothriuridae y es especialmente conspicua en machos.

Hábitos peri domiciliarios: Se refiere a la tendencia de algunos animales a vivir en la cercanía o periferia de construcciones humanas.

Hábitos sinantrópicos: Se refiere a los animales que viven en estrecha asociación con los seres humanos. En el caso de los escorpiones hacemos referencia a aquellos que viven dentro de la vivienda humana.

Introducción antropogénica: Se refiere a la introducción de una especie no nativa por la acción directa del ser humano.

Metasoma: También denominado cola. Es la parte final del cuerpo del escorpión (Segmentos 15 a 19). En el último segmento se encuentra el telson, el cual está conformado por la vesícula y el aguijón.

Mesosoma: Segunda parte del cuerpo. Se encuentra antes del metasoma, a diferencia del prosoma, éste se encuentra segmentado (Segmentos 8 a 14).

Partenogénesis: Es un tipo de reproducción basada en el desarrollo de células sexuales femeninas no fecundadas. En el caso de las especies presentes en Uruguay, la partenogénesis podría ser del tipo telítoca (la descendencia es exclusivamente femenina).

Pedipalpos: Segundo par de apéndices prosomáticos. Están formados por seis artejos, de los cuales los últimos dos (tibia y tarso) forman las pinzas o quelas. De éstas el tarso (inferior) es móvil, mientras que la tibia (superior) es inmóvil.

Prosoma: Se designa de esta forma a la primera parte del cuerpo. No se encuentra segmentado (Segmentos 1 a 6).
Quelíceros: Primer par de apéndices prosomáticos que pueden extenderlos hacia delante, utilizándolos para sujetar y cortar a las presas. Están formados por tres artejos -triarticulados-, el primero fijo y los dos restantes móviles.

Telson: Estructura ubicada en la parte final del metasoma o cola y formada por la vesícula y el aguijón.

\section{Reconocimientos}

Un sincero agradecimiento a Fernando Costa, Fernando PérezMiles y Miguel Simó por su continuo apoyo y sugerencias durante la confección de esta clave. A Anita Aisenberg por sus comentarios y sugerencias y particularmente a los revisores que mejoraron sustancialmente la presentación de la Clave. Las Figuras 5, 9 y 11 son cortesía de Laura Watson, la Figura 14 es cortesía de Mario Lalinde.

\section{Referencias}

- ACOSTA, L.E. Contribución al conocimiento taxonómico del género Urophonius Pocock, 1893 (Scorpiones, Bothriuridae). En: The Journal of Arachnology. 1988, 16:23-33.

- BENAMÚ, M.A. Clave para la determinación de algunas familias de arañas (Araneae, araneomorphae) de Uruguay. En: Boletín de la Sociedad Zoológica del Uruguay. 2007, 2da. época (16):1-19.

- BENTON, T.G. The life history of Euscorpius flavicaudis (Scorpiones, Chactidae) En: The Journal of Arachnology. 1991, 19:105-110.

- BENTON, T.G. The reproductive ecology of Euscorpius flavicaudis in England. En: Memoirs of the Queensland Museum. 1993, 33(2):445-460.

- COSTA, F.G.; PÉREZ-MILES, F. Ecología de los escorpiones Bothriuridae de Sierra de las Ánimas, Maldonado, Uruguay. En: Aracnología. 1994, 21:1-5.

- FET, V.; SISSOM, W.D.; LOWE, G.; BRAUNWALDER, M. Catalog of the scorpions of the world (1758-1998). New York: New York Entomological Society, 2000.

- FLÓREZ, E.D. Escorpiones de la familia Buthidae (Chelicerata: Scorpiones) de Colombia. En: Biota Colombiana. 2001, 2(1):2530.

- GIULIANI, L.; SIMÓ, M.; ACOSTA, L. Orden Opiliones. En: VIERA, C. Arácnidos del Uruguay: diversidad, comportamiento y ecología. Montevideo: Banda Oriental, 2011. pp. 79-90.

- HEUHS, L.; POSE, D.; GONZALEZ, S.; DELFINO, N. Accidentes por escorpiones en Uruguay. En: II Congreso Uruguayo de Toxicología Clínica y I Encuentro del Conosur sobre antídotos. Montevideo: [s.n.], 2002. p.25.

- LOURENÇO, W.R. Can a Bothriurid scorpion be present in the Himalayas of India. En: Entomologische Mitteilungen deer Zoologische Museum Hamburg. 1996, 12(154): 83-90.

- LOURENÇO, W.R.; MAURY, E.A. Contribution à la connaissance systématique Scorpions appartenant au «complexe» Tityus bolivianus Kraepelin, 1895 (Scorpiones, Buthidae). En: Revue Arachnologique. 1985, 6(3):107-126.

- LOURENÇO, W.R.; MONOD, L. Description of a new genus and species of scorpion (Bothriuridae) from Brazil. En: Ekologia. 2000, 19(3):145-152.

- MATTONI, C.I. Patrones evolutivos en el género Bothriurus (Scorpiones, Bothriuridae): Análisis filogenético. Córdoba: 
Facultad de Ciencias Exactas, Físicas y Naturales, 2003. (Tesis de Doctorado).

- MAURY, E.A. Aportes al conocimiento de los escorpiones de la República Argentina I. Observaciones biológicas sobre Urophonius brachycentrus (Thorell, 1877) (Bothriuridae). En: Physis. 1968, 27(75):407-418.

- MAURY, E.A. Observaciones sobre el ciclo reproductivo de Urophonius brachycentrus (Thorell, 1877) (Scorpiones, Bothriuridae). En: Physis. 1969, 29(78):131-139.

- MAURY, E.A. Tityus trivittatus en la Argentina nuevos datos sobre distribución, partenogénesis, sinantropía y peligrosidad (Scorpiones, Buthidae). En: Revista del Museo Argentino de Ciencias Naturales “Bernardino Rivadavia”. 1997, 24:1-24.

- MONTES DE OCA, L.; PÉREZ-MILES, F. Las arañas Mygalomorphae del Uruguay: clave para familias, géneros y especies. En: INNOTEC. 2009, (4):41-49.

- OJANGUREN-AFFILASTRO, A.A. Estudio monográfico de los escorpiones de la República Argentina. En: Revista Ibérica de Aracnología. 2005. 75-241.

- PERETTI, A.V. Relación de las glándulas caudales de machos de escorpiones Bothriuridae con el comportamiento sexual (Scorpiones). En: Revue Arachnologique. 1997, 12 (3):31-41.

- PRENDINI, L. A new genus and species of bothriurid scorpion from the Brandberg Massif, Namibia, with a reanalysis of bothriurid phylogeny and a discussion on the phylogenetic position of Lisposoma Lawrence. En: Systematic Entomology. 2003, 28:149-172.

- SAN MARTÍN, P.R. Observaciones sobre la ecología y distribución geográfica de tres especies de escorpiones en el Uruguay. En: Revista de la Facultad de Humanidades y Ciencias. 1961, 19:175-212.

- SAN MARTÍN, P.R. Una nueva especie de Bothriurus (Scorpiones, Bothiruridae) del Uruguay. En: Bulletin du Muséum National D'Histoire Naturelle. 1963, 2ª . Serie, 35 (4):400-418.

- SAN MARTÍN, P.R. Escorpiofauna Uruguaya II Bothriurus rochensis, nueva especie de Bothriuridae del Uruguay. En: Comunicaciones Zoológicas del Museo de Historia Natural de Montevideo. 1965, VIII(106):1-22.

- SAN MARTÍN, P.R; L.A. de GAMBARDELLA. Contribución a la ecología de los escorpiones (Bothriuridae). Hábitat de tres especies de Bothriurus del Uruguay y su aplicación en la sistemática. En: Bulletin du Muséum National D'Histoire Naturelle. 1967, 39(1):188-196.

- TOSCANO-GADEA, C.A. Escorpiofauna de los Bañados del Este II Departamento de Rocha, Uruguay. En: Encuentro de Jóvenes Biólogos. Montevideo: Facultad de Ciencias, 1996. p.136-137.

- TOSCANO-GADEA, C. A. Euscorpius flavicaudis (Degeer, 1778) in Uruguay: first record from the new world. En: Newsletter of the British Arachnological Society. 1998, 81:6.

- TOSCANO-GADEA, C. A. Is really Tityus uruguayensis (Scorpionida, Buthidae) parthenogentic? En: FET, Victor; SELDEN, Paul. Scorpions 2001: In Memoriam of Gary A. Polis. London: British Arachnological Society, 2001. pp.359-364.

- TOSCANO-GADEA, C.A. Fenología y distribución de la escorpiofauna del Cerro de Montevideo, Uruguay: un estudio de dos años con trampas de caída. En: Revista Ibérica de Aracnología. 2002, 5:77-82.

- TOSCANO-GADEA, C.A. Confirmation of parthenogenesis in Tityus trivittatus Kraepelin 1898 (Scorpiones, Buthidae). En: The Journal of Arachnology. 2005, 32:866-869.
- TOSCANO-GADEA, C. A. Nuevos aportes para una vieja controversia: ¿Es Tityus uruguayensis (Scorpiones, Buthidae) realmente partenogenético? En: Actas de las IX Jornadas de Zoología del Uruguay, Facultad de Ciencias. Montevideo: FCIEN, 2008. pp. 75.

- TOSCANO-GADEA, C.A. Escorpiones en el Uruguay. En: AISENBERG, A.; TOSCANO-GADEA, C.A.; GHIONE, S. Guía de arácnidos del Uruguay. Montevideo: MEC, 2011a pp.196-199.

- TOSCANO-GADEA, C.A. Los escorpiones en Uruguay: pasado, presente y futuro. En: VIERA, C. Arácnidos del Uruguay: diversidad, comportamiento y ecología. Montevideo: Banda Oriental, 2011b. pp. 51-76.

- ZOLESSI, L. C. DE. La partenogénesis en el escorpión amarillo Tityus uruguayensis (Borelli, 1900) (Scorpionida: Buthidae). En: Revista de la Facultad de Humanidades y Ciencias. 1985, 3ra. época 1:25-32. 